\title{
Modelización del efecto de los cambios de uso del suelo sobre los flujos de nutrientes en cuencas agrícolas costeras: el caso del Mar Menor (Sudeste de España)
}

\author{
(1) Departamento de Biología Aplicada. Universidad Miguel Hernández de Elche \\ (2) Soil and Water Science Department. University of Florida \\ (3) Departamento de Ecología e Hidrología. Universidad de Murcia \\ (4) Institute for Environment and Sustainability. Joint Research Center \\ *Autor de correpondencia: J.Martínez Fernández [maria.martinezf@umh.es]
}

J.Martínez Fernández ${ }^{1, *}$, C. Fitz², M.A. Esteve Selma³, N. Guaita², J. Martínez-López

> Recibido el 10 de noviembre de 2013, aceptado el 16 de diciembre de 2013.

\begin{abstract}
Martínez Fernández, J., Fitz, C., Esteve Selma, M.A., Guaita, N., Martínez-López, J. 2013. Modelización del efecto de los cambios de uso del suelo sobre los flujos de nutrientes en cuencas agrícolas costeras: el caso del Mar Menor (Sudeste de España). Ecosistemas 22(3):84-94. Doi.: 10.7818/ECOS.2013.22-3.12.

Modelización del efecto de los cambios de uso sobre los flujos de nutrientes en cuencas agrícolas costeras: el caso del Mar Menor (Sudeste de España). Se ha elaborado un enfoque integrado de modelización de la dinámica hidrológica de una cuenca agrícola, la cuenca de la laguna costera del Mar Menor (Sudeste de España), para analizar los efectos de los cambios de uso a medio y largo plazo sobre los flujos de nutrientes que alcanzan la laguna y sus humedales periféricos. Se ha elaborado un modelo socioambiental sin dimensión espacial explícita que presta especial atención a los factores socio-económicos y los cambios de uso. Se presentan también los resultados preliminares de la aplicación al Mar Menor del modelo ELM (Ecological Landscape Modeling), un modelo hidroecológico con dimensión espacial explícita, como mejora de la modelización hidrológica de la cuenca. Los resultados del modelo integrado (1970-2012) muestran el significativo incremento de la entrada de nutrientes en la laguna del Mar Menor, la existencia de grandes fluctuaciones interanuales y el papel de los humedales en la retención de una parte de tales nutrientes. Se ha aplicado el modelo para analizar los efectos previsibles de distintas medidas de gestión orientadas a reducir la exportación de nutrientes a la laguna del Mar Menor, en particular la reutilización de drenajes agrícolas y la recuperación de humedales. Los resultados de la simulación de tales medidas señalan que la recuperación de humedales es más eficaz que la reutilización de drenajes, consiguiendo una reducción más significativa de los nutrientes que finalmente alcanzan el Mar Menor.
\end{abstract}

Palabras clave: modelo socio-ambiental; regadío; simulación dinámica, ciclos de nutrientes

Martínez Fernández, J., Fitz, C., Esteve Selma, M.A., Guaita, N., Martínez-López, J. 2013. Modelling the effects of land use change on the nutrient dynamics in a coastal agricultural watershed: the Mar Menor case (Southeastern Spain). Ecosistemas 22(3):84-94. Doi.: 10.7818/ECOS.2013.22-3.12.

An integrated modelling approach on the nutrient dynamics has been developed for its application in the agricultural watershed of the Mar Menor coastal lagoon (South-eastern Spain), in order to analyse the long-term effects of land-use change on the nutrients inputs into the lagoon and associated wetlands. A lumped dynamic model has been developed which focuses on the socio-economic factors and land-use changes and their effects on the watershed-scale nutrient flows. In addition, a preliminary application of the ELM (Ecological Landscape Model), an eco-hydrological spatial dynamic model, has been carried out as an ongoing improvement of the hydrological modelling module. Simulation results for the 1970-2012 period showed a noticeable increase in nutrient inflows into the Mar Menor lagoon, highlighting the high inter-annual variability and the role of associated wetlands to remove part of the nutrients. The modeling approach was applied to explore the potential effects of several management measures aiming at reducing the export of nutrients from the agricultural fields into the Mar Menor lagoon, particularly studying measures to reuse agricultural leakages and plans for wetlands restoration. Results showed that wetlands restoration achieves a higher reduction in the inflow of nutrients into the lagoon, doubling those achieved by reusing agricultural leakages.

Key words: dynamic simulation; irrigated lands; socio-environmental model, nutrient cycling

\section{Introducción}

En los últimos años se han desarrollado y aplicado distintas herramientas que permiten la modelización de la dinámica hidrológica a escala de cuenca, lo que sin duda está suponiendo un gran avance en la comprensión de dicha dinámica a nivel espacial y temporal. Pese a estos indudables avances, permanecen algunos retos pendientes, como la necesidad de abordar de forma más integral (en el sentido indicado por Kelly et al. 2013) las relaciones entre los factores sociales, económicos y ambientales del sistema socio ambiental más amplio, así como la de entender la forma en que los cambios de uso afectan a los ecosistemas y a los servicios que los mismos aportan (Galic et al. 2012) y la de evaluar cómo tales servicios ecosistémicos pueden cambiar bajo diferentes escenarios y estrategias de manejo. Abordar tales retos requiere i) atender los contextos específicos que se pretenden modelar, ii) considerar los 
procesos clave del sistema socio ambiental que gobierna la cuenca objeto de estudio, incluyendo los cambios de uso y iii) aplicar una perspectiva a medio y largo plazo.

Si bien la elaboración y aplicación de modelos puede contribuir a la comprensión de estos procesos y sus efectos con el fin de contribuir a una gestión adaptativa (Maltby et al. 2013), permanece insuficientemente abordada, particularmente en el caso de sistemas mediterráneos (EEA 2010), la modelización de los sistemas socioambientales dentro de los cuales tienen lugar los cambios de uso del suelo o decisiones de gestión (si bien pueden consultarse algunos ejemplos de modelos socio-ambientales en Martínez-Fernández et al. 2000, Martínez-Fernández et al 2004; Martínez-Fernández et al. 2013 y Vidal et al. 2013) y la forma en que tales cambios y decisiones afectan a la dinámica de los ecosistemas (Nemec y Raudsepp-Hearne 2013), reto igualmente pendiente en el caso de la dinámica integrada de las cuencas. En efecto, más allá de los efectos directos sobre el territorio, los cambios de uso a escala de cuenca, particularmente en el caso de cuencas agrícolas, generan importantes efectos indirectos a través de la alteración de los flujos hídricos y de nutrientes, los cuales inciden a su vez sobre los ecosistemas acuáticos (sistemas fluviales, ramblas, humedales, aguas costeras), los espacios naturales o la biodiversidad y sobre los servicios ecosistémicos que los mismos generan (Fig. 1). Anticipar los efectos previsibles de nuevos cambios de uso o de distintas opciones de gestión requiere disponer de modelos que integren componentes de muy distinta naturaleza y que conecten distintas escalas, como la de los humedales y la dinámica general de la cuenca de la que dependen.

Los humedales costeros mediterráneos proporcionan un amplio conjunto de servicios ecosistémicos (véase por ejemplo la revisión de EEA 2010), pese a lo cual se encuentran amenazados por múltiples factores y en particular por las principales tendencias de cambio de uso en el ámbito mediterráneo, en las que destaca la intensificación agrícola a través del regadío (Antrop 1993; de Aranzábal et al. 2008), lo que da lugar a importantes efectos sobre el funcionamiento hidrológico de las cuencas. Pese a ello, las relaciones entre el humedal y su cuenca se siguen ignorando en la gestión de la mayoría de estos ecosistemas (Houlahan y Findlay 2004), lo que supone un serio problema para su conservación. Por ejemplo, se estima que más de la mitad de los humedales mediterráneos se han perdido por drenaje, transformación agrícola, contaminación o sobreexplotación (IUCN 2002), siendo especialmente vulnerables los humedales costeros (EEA 2010). Urge por ello desarrollar y aplicar herramientas que faciliten la integración entre los humedales y sus cuencas, con el fin de prever los efectos sobre los humedales de distintos cambios de uso del suelo u opciones de manejo.

Como contribución a los retos indicados, se está desarrollando un enfoque integrado de modelización en la cuenca de la laguna costera del Mar Menor, (Sudeste de España), cuenca en la que distintos procesos y factores tanto socioeconómicos como ambientales están alterando los flujos de agua y nutrientes (véase por ejemplo Martínez-Fernández y Esteve Selma 2003; Conesa y Jiménez-Cárceles 2007) y afectando a la laguna del Mar Menor, sus humedales y comunidades biológicas asociadas (véase por ejemplo Carreño et al. 2008; Esteve et al. 2008; Pardo et al. 2008; Robledano et al. 2010, 2011).

Entre otros efectos, el incremento en la entrada de nutrientes a la laguna del Mar Menor ha modificado su estructura y dinámica. La concentración de nitrógeno en el agua de la laguna ha aumentado de forma significativa (Perez Ruzafa et al. 2002; Lloret et al. 2005; Velasco et al. 2006), de forma que en diez años dicha concentración aumentó en un orden de magnitud (Pérez Ruzafa et al. 2002), lo que ha generado múltiples efectos, como una gran producción primaria fitoplanctónica que a su vez ha favorecido, junto a otros factores, la proliferación estival de medusas en la laguna del Mar Menor (Perez-Ruzafa et al. 2002, Lloret et al. 2005; Conesa y Jiménez-Cárceles 2007; Lloret et al. 2008). El aumento de los flujos de agua y nutrientes de la cuenca han dado lugar también a importantes cambios en los humedales litorales del Mar Menor así

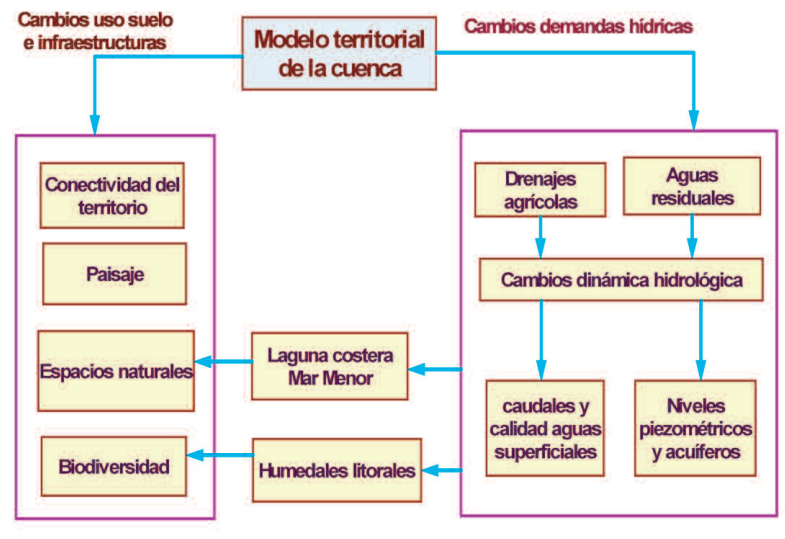

Figura 1. Efectos directos e indirectos del modelo territorial de la cuenca sobre distintos componentes de la misma y sus sistemas naturales.

como en distintas comunidades como las comunidades vegetales, las aves acuáticas, las aves esteparias y los coleópteros (Esteve et al. 2008). A lo anterior se añade el hecho de que la necesidad de reducir la entrada de nutrientes constituye también una obligación legal derivada de la Directiva Marco de Agua así como de las directivas europeas de Nitratos (91/676 ECC) y sobre aguas residuales (91/271 ECC).

En el contexto de un enfoque integrado, actualmente en desarrollo, este trabajo presenta la modelización de la cuenca, con los siguientes objetivos específicos: i) integrar los factores socioeconómicos y ambientales clave que gobiernan la dinámica de los nutrientes a escala de cuenca; ii) comprender el efecto de los cambios de uso del suelo y el papel de los humedales en dicha dinámica y iii) utilizar dicho enfoque integrado de modelización para explorar los efectos potenciales de algunas opciones de gestión actualmente en discusión.

\section{Materiales y métodos}

\section{Area de estudio}

Con unos $135 \mathrm{~km}^{2}$ de superficie, el Mar Menor es la mayor laguna costera del Mediterráneo Occidental. El Mar Menor sustenta importantes valores ecológicos y naturales (humedales litorales, avifauna, hábitats y otros) refrendados con diversas figuras de protección a nivel nacional e internacional (espacio protegido, Zona de Especial Protección para las Aves, Lugar de Importancia Comunitaria, área Ramsar y Zona Especialmente Protegida de Importancia para el Mediterráneo). La cuenca del Mar Menor (lat $=37,1 \mathrm{~N}$, long $=-1 \mathrm{~W}$ ) constituye una extensa llanura de unos $1200 \mathrm{~km}^{2}$ drenada por un conjunto de ramblas que desembocan en la laguna. El área de estudio tiene un clima mediterráneo semiárido, con una temperatura media anual de unos $17^{\circ} \mathrm{C}$ y una precipitación media anual en torno a $330 \mathrm{~mm}$, con una gran variación interanual.

Como en otras cuencas mediterráneas, los episodios de precipitaciones intensas y avenidas juegan u papel importante (David et al. 1997; Xue et al. 1998). El papel crucial de las avenidas se sustenta en otras evidencias, como datos empíricos de concentración de nutrientes en las ramblas que desembocan en el Mar Menor durante los episodios de avenida (Velasco et al. 2006, Alvarez Rogel et al. 2009; Serrano y Sironi 2009), en los que no sólo aumenta espectacularmente el volumen de agua que llega a la laguna sino que también se incrementa la concentración de nutrientes contenida en la misma. De la misma forma, los datos de concentración de contaminantes orgánicos disueltos transportados por la rambla principal (rambla del Albujón) aumentan en varios órdenes de magnitud durante episodios de precipitaciones intensas y avenidas, las cuales son responsables de más del $70 \%$ de la entrada total de pesticidas desde dicha rambla (Moreno-González et al. 2013).

Actualmente la principal actividad económica en la cuenca es el regadío intensivo (Fig. 2). Hasta hace tres décadas el regadío era bastante limitado, dada la escasez de recursos hídricos, pero 


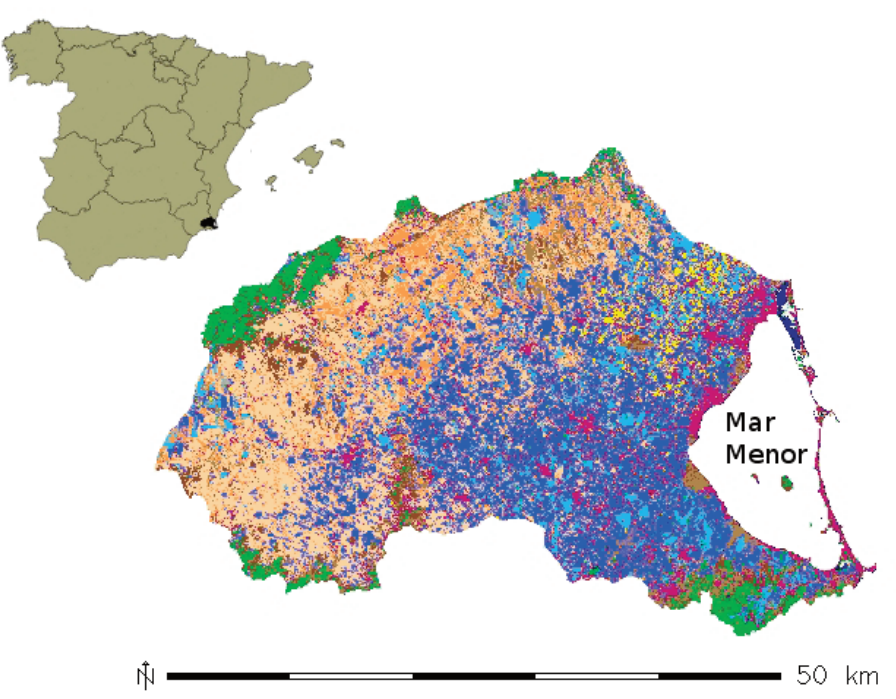

Figura 2. Localización y principales usos del suelo en la cuenca del Mar Menor. Verde: natural; azul: regadío al aire libre; amarillo: invernaderos; crema: secano; granate: urbano e infraestructuras.

la llegada de las aguas del trasvase Tajo-Segura en 1979 supuso una profunda transformación de la cuenca, con un importante incremento del regadío, especialmente de los invernaderos y sobre todo del regadío hortícola al aire libre, que alcanza una expansión muy notable. El incremento del regadío supuso un gran aumento de la aportación de flujos hídricos y de nutrientes a la laguna y humedales periféricos (Velasco et al. 2006; Carreño et al. 2008, Esteve et al. 2008). Por otra parte en el entorno más próximo a la laguna, pese al mantenimiento de cierta actividad pesquera y salinera, la principal actividad es la turística, sobre todo estival, lo que contribuye también a la aportación de nutrientes.

\section{Marco metodológico general}

Se está desarrollando y aplicando un enfoque integrado para modelar y analizar la dinámica hídrica y de nutrientes de la cuenca, los factores que gobiernan los cambios de uso a largo plazo en la cuenca, los efectos de dichos cambios sobre los flujos de nutrientes, el papel de los humedales litorales y en qué medida los cambios observados en la laguna del Mar Menor (como la proliferación estival de medusas), en los humedales litorales (cambios en la vegetación y hábitats naturales) y en distintas comunidades faunísticas (aves acuáticas, aves esteparias, coleópteros errantes) responden a las transformaciones sufridas en la cuenca, entre otros factores (Esteve et al. 2008). Este carácter integrado se refiere a la inclusión de los factores socio-económicos y ambientales dentro de un mismo modelo, lo que permite abordar las interacciones entre los mismos, así como al uso integrado de distintas herramientas, como los modelos de simulación, el análisis de escenarios y el análisis coste-efectividad. Dicho enfoque integrado ha permitido además una primera aproximación a la valoración de algunos de los servicios ecosistémicos, como la retención de nutrientes por parte de los humedales, a la identificación de las relaciones entre la conservación de los espacios protegidos del Mar Menor y la gestión que se realiza a otras escalas, como la cuenca, así como a la detección de contradicciones potenciales entre distintos objetivos ambientales o servicios ecosistémicos, por ejemplo entre la conservación de la biodiversidad de los humedales, especialmente de comunidades y hábitats singulares adaptados a humedales en ambientes semiáridos, afectados negativamente por el aumento de los flujos y la función de retención de nutrientes de estos humedales, cuya maximización requeriría el incremento de tales flujos (Martínez Fernández et al. en prensa).

El presente trabajo se centra en la modelización de la cuenca y de los efectos de los cambios de uso sobre los flujos de nutrientes utilizando un modelo socioambiental integrado, sin dimensión espacial explícita, con particular atención a los factores socio-económicos, los cambios de uso del suelo y las dinámicas a largo plazo de los flujos de nutrientes, si bien se presentan también algunos resultados preliminares de la aplicación del modelo ELM (Ecological Landscape Modeling) a la cuenca del Mar Menor, como mejora de la modelización hidrológica de la cuenca, actualmente en fase de desarrollo. ELM es un modelo dinámico espacialmente explícito, con particular atención a la integración de los procesos espaciales y temporales de los flujos de agua y nutrientes, lo que permitirá un análisis más detallado a nivel espacial, de especial importancia de cara a la gestión.

\section{El modelo socioambiental integrado de la cuenca del Mar Menor}

Se ha elaborado un modelo socioambiental de la cuenca del Mar Menor que incorpora los factores ambientales y socioeconómicos implicados en la exportación de nutrientes a la laguna del Mar Menor (Fig. 3). Como horizonte temporal se ha considerado el periodo 1970-2012, para recoger la dinámica a largo plazo y en particular los cambios introducidos por la llegada en 1979 de las aguas del trasvase Tajo-Segura y sus efectos sobre los cambios de uso del suelo. El modelo tiene en cuenta el importante impacto de los episodios de avenida sobre la dinámica hidrológica y sobre la movilización de nutrientes en cuencas mediterráneas. Se ha utilizado un modelo hidrológico (Chapelle et al. 2005) para estimar la escorrentía superficial y subsuperficial procedentes de las precipitaciones y de los drenajes de riego. Este modelo hidrológico genera series temporales de escorrentía que son posteriormente utilizadas en el modelo dinámico. El modelo socioambiental integrado del Mar Menor es un modelo dinámico sin dimensión espacial explícita que utiliza una resolución temporal diaria y realiza simulaciones largas (varias décadas) para captar la dinámica a largo plazo del sistema. El modelo incluye varios sectores: i) sector del nitrógeno; ii) sector del fósforo iii) usos del suelo; iv) las salmueras generadas por las desalobradoras del Campo de Cartagena; v) los humedales asociados al Mar Menor vi) sector urbano y vi) los costes económicos asociados a diversas medidas de gestión. Todos los sectores del modelo están interconectados a través de distintas variables. Las principales salidas del modelo son las siguientes: Series diarias a largo plazo (1970-2012) de la superficie ocupada por los principales usos del suelo, población residente y estival del entorno del Mar Menor, flujos de nitrógeno y de fósforo procedentes de las áreas agrícolas de la cuenca y de las aglomeraciones urbanas y entrada final de nitrógeno y fósforo que alcanza la laguna del Mar Menor.

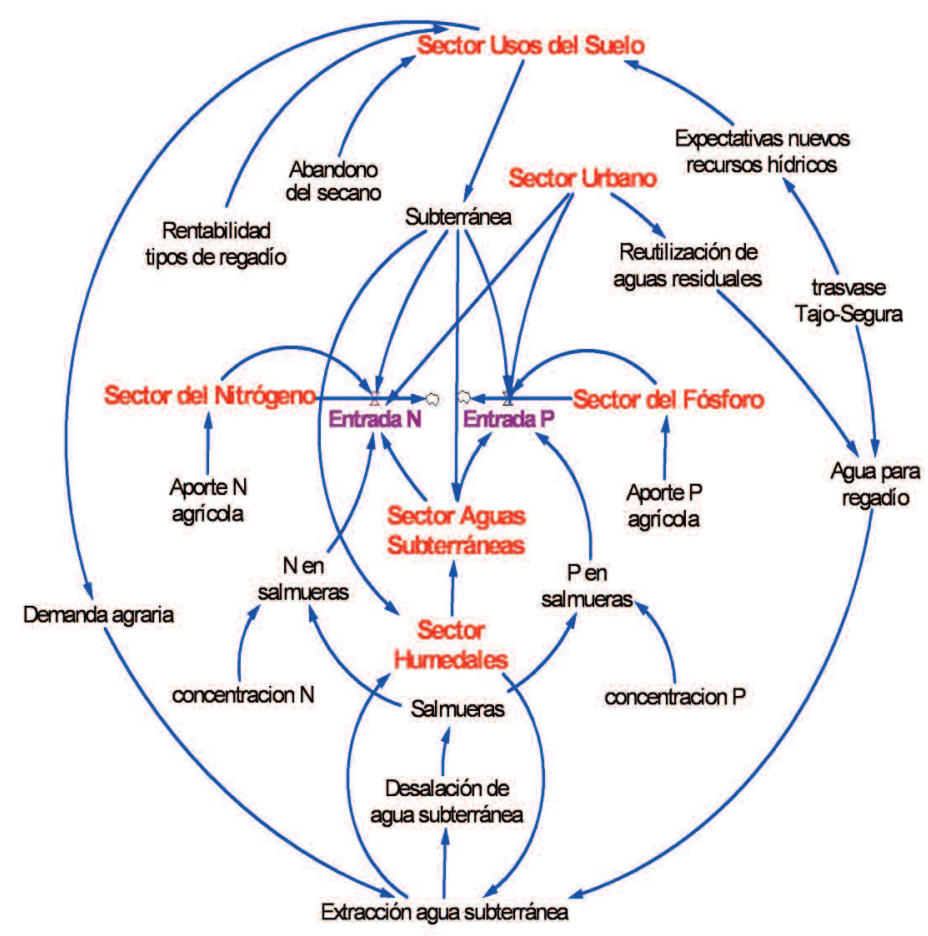

Figura 3. Diagrama simplificado del modelo dinámico de la cuenca del Mar Menor 
El Sector del Nitrógeno (Fig. 4) tiene en cuenta los contenidos en nitrógeno de la solución del suelo, la hojarasca y la vegetación, así como los flujos entre estos compartimentos. El sector del Fósforo considera los correspondientes flujos y compartimentos, similares a los del nitrógeno. El Sector de los Humedales se centra en la funcionalidad de estos sistemas para retener y eliminar una parte de los nutrientes procedentes de la cuenca. Las principales variables del Sector de los Humedales se refieren a la superficie de humedal activo, la capacidad de retención de nitrógeno y fósforo de cada humedal y el efecto del caudal sobre la retención de nutrientes.

Los factores socioeconómicos no están considerados en un modelo separado sino que constituyen parte de las variables del modelo integrado, en estrecha interacción con las variables ambientales. El sector de Usos del Suelo (Fig. 5) considera los cambios de uso entre la superficie ocupada por vegetación natural el secano, el regadío arbóreo, el regadío herbáceo al aire libre, los invernaderos y las zonas urbanas. Cada uso del suelo presenta características específicas en relación con la cantidad y dinámica del nitrógeno y el fósforo, por lo que los cambios de uso afectan notablemente a los sectores de los nutrientes. El sector de usos del suelo permite simular los principales cambios de uso a escala de cuenca inducidos por diversos factores socio-económicos, en particular el incremento del regadío, producido como consecuencia de la llegada de las aguas del trasvase Tajo- Segura en 1979 y la mayor rentabilidad de los cultivos de regadío frente al secano. El Sector Urbano tiene en cuenta la evolución de la población residente y la población turística estacional, el volumen de aguas residuales, la eficiencia de las depuradoras, el grado de reutilización de las aguas residuales en el uso agrícola, y los vertidos urbanos finales. Por último el modelo incluye un sector de Costes, que permite la realización de un análisis coste-efectividad de las principales medidas de gestión.

El modelo dinámico ha sido calibrado manualmente utilizando los datos disponibles del periodo 1970-2012, mayoritariamente relativos a la superficie ocupada por cada uso del suelo (natural, secano, regadío arbóreo, regadío herbáceo al aire libre e invernaderos), procedentes de teledetección y del Servicio de Estadística de la Región de Murcia, la población residente y la población estival del entorno del Mar Menor (Servicio de Estadística de la Región de Murcia) y, de forma mucho más escasa, datos parciales sobre caudales y concentraciones de algunos flujos (SACYR 1997; Lloret et al. 2005; Velasco et al. 2006, García Pintado et al. 2009, Serrano y Sironi 2009; Alvarez Rogel et al. 2009). Dada la escasez de datos observados y el enfoque de largo plazo del modelo, se ha optado por utilizar todos los datos disponibles para su calibración. Los datos para la estimación de parámetros proceden de una amplia variedad de fuentes, incluyendo trabajo de campo, bases de datos estadísticos y literatura científica (Tabla 1). Los parámetros relativos a los aportes de fertilizantes y coeficientes de lixiviado desde las parcelas agrícolas proceden de estudios empíricos del campo del Mar Menor, que han resultado próximos a los encontrados en otros regadíos mediterráneos intensivos (Guimerà et al. 1995; Moreno et al. 1996). El sector de los humedales ha sido parametrizado con trabajo de campo específico realizado en los humedales litorales del Mar Menor y aplicando modelos de regresión para estimar la retención de nutrientes en función de la longitud de los cauces a lo largo del humedal y en función del caudal. El sector de los usos del suelo se ha desarrollado y parametrizado a partir de teledetección y clasificación supervisada. La mayor parte de los datos socioeconómicos proceden de servidores estadísticos regionales (Centro Regional de Estadística de la Región de Murcia y Servicio de Estadística Agraria de la Región de Murcia)

\section{Aplicación preliminar del entorno ELM a la cuenca del Mar Menor}

Aunque este trabajo se centra en la dinámica socioambiental a largo plazo, utilizando el modelo integrado descrito en el apartado anterior, es importante aplicar herramientas de modelado dinámico espacialmente explícitas que permitan comprender y gestionar los

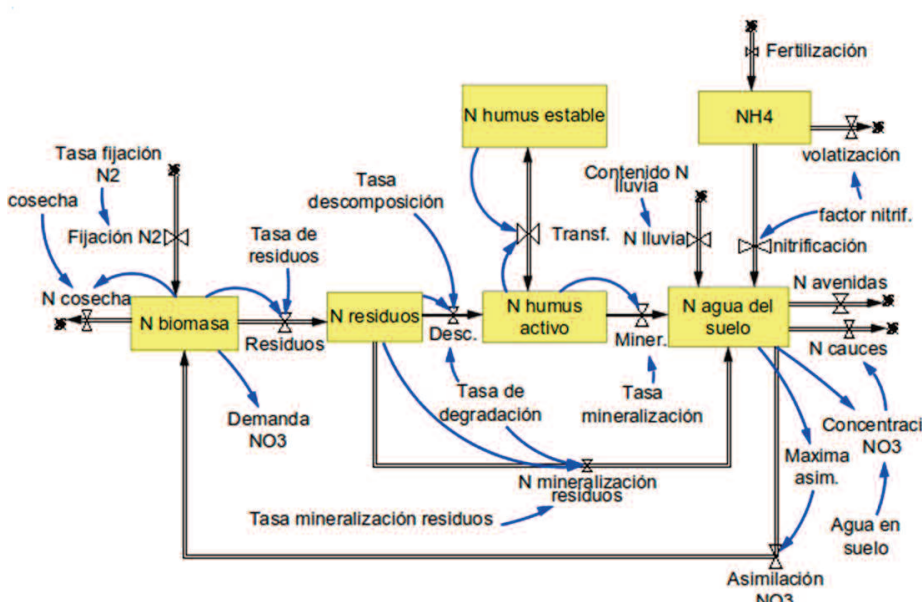

Figura 4. Diagrama simplificado del sector del Nitrógeno

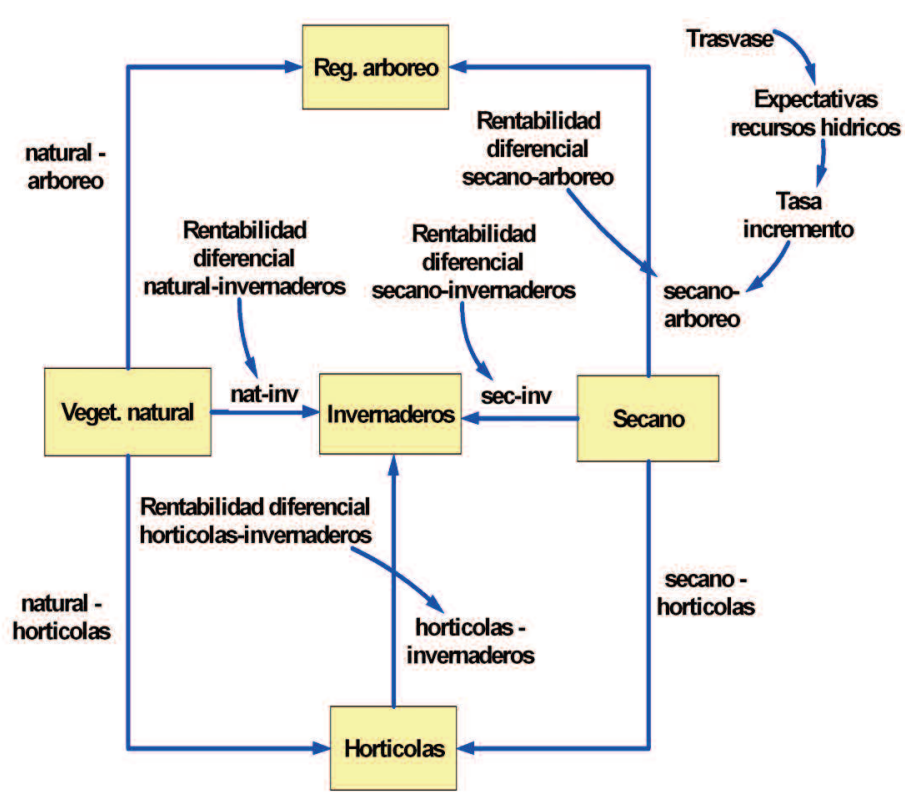

Figura 5. Diagrama simplificado del sector de los Usos del Suelo

flujos de nutrientes a escalas más detalladas. Por ello actualmente está en fase de desarrollo la mejora de la modelización hidrológica de la cuenca del Mar Menor a través de un modelo dinámico espacialmente explícito, utilizando el entorno ELM (Ecological Landscape Modeling), cuyos resultados preliminares se presentan en el apartado correspondiente. ELM es una herramienta de modelización que permite la elaboración de modelos hidro-ecológicos dinámicos y espacialmente distribuidos (Fitz 2008, Fitz et al. 2011), la cual ha sido aplicada para comprender y restaurar el sistema de las Everglades, en Florida, USA (pueden consultarse detalles de las interacciones entre ciencia y gestión y la aplicación del modelo ELM a las Everglades en NRC 2012). Se trata de un modelo dinámico espacial que integra la hidrología superficial y subterránea, la salinidad, la dinámica del nitrógeno y la del fósforo teniendo en cuenta los distintos compartimentos: tipos de vegetación, suelo, flujos subsuperficiales y cuerpos de agua. Las principales salidas del modelo incluyen la escorrentía superficial, la percolación hacia el acuífero, los niveles piezométricos y los flujos de nitrógeno y fósforo. (pueden consultarse detalles en http://ecolandmod.ifas.ufl.edu; Fitz y Paudel 2012). El entorno ELM fue diseñado con el suficiente grado de generalidad y flexibilidad que permitiera la comprensión de la dinámica integrada de los ecosistemas en dominios espaciales y temporales amplios (Fitz 2010). Es un proyecto de código abierto (Open Source).

Una vez se complete la aplicación del entorno ELM al Mar Menor, actualmente en fase de desarrollo, se conectarán ambos 
Tabla 1. Principales parámetros del modelo socioambiental integrado de la cuenca del Mar Menor

\begin{tabular}{|c|c|c|c|c|}
\hline Parámetro & Definición & Valor & Unidades & Fuente \\
\hline$d w n$ & Biomasa (peso seco) por hectárea en las áreas de vegetación natural & 14100 & $\mathrm{~kg} / \mathrm{ha}$ & $\begin{array}{l}\text { A partir de Martínez Fernández et al } \\
(1994,1995,1996)\end{array}$ \\
\hline$d w d$ & Biomasa (peso seco) por hectárea en los cultivos de secano & 5625 & $\mathrm{~kg} / \mathrm{ha}$ & $\begin{array}{l}\text { Consejería de Agricultura. Comunidad } \\
\text { Autónoma de la Región Murcia }\end{array}$ \\
\hline nir & Aportación de nitrógeno con la precipitación & 0.0959 & $\mathrm{~kg} /$ (ha.día) & Gómez Cerezo (1995) \\
\hline$n h 4 d f$ & Aportación media de N-NH4 en la fertilización en secano & 0.01 & $\mathrm{~kg} /$ (ha.día) & Olmo Conesa (2004) \\
\hline nh4if & Aportación media de N-NH4 en la fertilización en regadío & 0.05 & $\mathrm{~kg} /($ ha.día) & Olmo Conesa (2004) \\
\hline pitf & Aportación media de $\mathrm{P}$ en la fertilización en regadío arbóreo & 0.0384 & $\mathrm{~kg} /$ (ha.día) & Olmo Conesa (2004) \\
\hline phf & Aportación media de $\mathrm{P}$ en regadío herbáceo al aire libre & 0.243 & $\mathrm{~kg} /$ (ha.día) & Olmo Conesa (2004) \\
\hline pgf & Aportación media de $\mathrm{P}$ en la fertilización en invernaderos & 0.934 & $\mathrm{~kg} /$ (ha.día) & Olmo Conesa (2004) \\
\hline$n n b$ & Contenido medio de nitrógeno en la biomasa de la vegetación natural & 0.0153 & s.d. & Castro Diez (1996) \\
\hline$n d b$ & Contenido medio de nitrógeno en la biomasa de los cultivos de secano & 0.0231 & s.d. & $\begin{array}{l}\text { A partir de Rubio López (1999) y Shen et } \\
\text { al (2004) }\end{array}$ \\
\hline nitb & Contenido medio de nitrógeno en la biomasa del regadío arbóreo & 0.0136 & s.d. & Matos et al (2003) \\
\hline$n d r$ & Contenido medio de nitrógeno en residuos de cultivos de secano & 0.005 & s.d & $\begin{array}{l}\text { A partir de Rubio López (1999) y Shen et } \\
\text { al (2004) }\end{array}$ \\
\hline nitr & Contenido medio de nitrógeno en residuos de regadío arbóreo & 0.0042 & s.d. & Matos et al (2003) \\
\hline nprr & Relación P:N en residuos & 0.035 & s.d. & $\begin{array}{l}\text { A partir de Matos et al (2003), Shah et al } \\
\text { (2003) y Shen et al (2004) }\end{array}$ \\
\hline$w f c$ & Contenido volumétrico de agua en el suelo a capacidad de campo & 51.78 & $\mathrm{~mm}$ & Ramírez Santigosa et al (1999) \\
\hline$w w p$ & Contenido volumétrico de agua en el suelo a punto de marchitamiento & 27.46 & $\mathrm{~mm}$ & Ramírez Santigosa et al (1999) \\
\hline$s d$ & Densidad media del suelo & 1468 & $\mathrm{~kg} / \mathrm{m}^{3}$ & Ramírez Santigosa et al (1999) \\
\hline$s p$ & Porosidad media del suelo & 0.4458 & s.d. & Ramírez Santigosa et al (1999) \\
\hline gnrc & Coeficiente retención $\mathrm{N}$ en flujos subterráneos en los humedales & 0.99 & s.d. & Vidal-Abarca y Esteve Selma (1998) \\
\hline gprc & Coeficiente retención $\mathrm{P}$ en flujos subterráneos en los humedales & 0.8 & s.d. & Vidal-Abarca y Esteve Selma (1998) \\
\hline$d e c$ & $\begin{array}{l}\text { Eficiencia (relación agua producto/agua bruta) de las estaciones de } \\
\text { desalobración }\end{array}$ & 0.6 & s.d. & Vidal-Abarca y Esteve Selma (1998) \\
\hline$n c d$ & Contenido medio de nitrógeno en los salmueroductos de la cuenca & 0.06972 & $\mathrm{~kg} / \mathrm{m}^{3}$ & Vidal-Abarca y Esteve Selma (1998) \\
\hline pcd & Contenido medio de fósforo en los salmueroductos de la cuenca & 0.00116 & $\mathrm{~kg} / \mathrm{m}^{3}$ & Vidal-Abarca y Esteve Selma (1998) \\
\hline wpc & Generación de agua residual per cápita en el entorno del Mar Menor & 0.175 & $\mathrm{~m}^{3} /($ hab.día) & ) Ayuntamiento de Cartagena \\
\hline$n c w$ & Contenido medio de nitrógeno en las aguas residuales & 0.07135 & $\mathrm{~kg} / \mathrm{m}^{3}$ & $\begin{array}{l}\text { Dirección General del Agua. Consejería } \\
\text { de Agricultura, Agua y Medio Ambiente }\end{array}$ \\
\hline pcw & Contenido medio de fósforo en las aguas residuales & 0.00995 & $\mathrm{~kg} / \mathrm{m}^{3}$ & $\begin{array}{l}\text { Dirección General del Agua. Consejería } \\
\text { de Agricultura, Agua y Medio Ambiente }\end{array}$ \\
\hline npe & Eficiencia media de depuración para el nitrógeno en las depuradoras & 0.4008 & s.d. & $\begin{array}{l}\text { Dirección General del Agua. Consejería } \\
\text { de Agricultura, Agua y Medio Ambiente }\end{array}$ \\
\hline ppe & Eficiencia media de depuración para el fósforo en las depuradoras & 0.5038 & s.d. & $\begin{array}{l}\text { Dirección General del Agua. Consejería } \\
\text { de Agricultura, Agua y Medio Ambiente }\end{array}$ \\
\hline gavh & VAB (Valor Añadido Bruto) medio del regadío hortícola & 10600 & $€ /$ ha & $\begin{array}{l}\text { Servicio de Estadística de la Región de } \\
\text { Murcia }\end{array}$ \\
\hline gavg & VAB medio del cultivo en invernadero & 39774 & $€ /$ ha & $\begin{array}{l}\text { Servicio de Estadística de la Región de } \\
\text { Murcia }\end{array}$ \\
\hline gavt & VAB medio per cápita de las actividades turísticas & 1011 & $€ /$ turista & $\begin{array}{l}\text { Ayuntamiento de San Javier y Servicio de } \\
\text { Estadística de la Región de Murcia }\end{array}$ \\
\hline
\end{tabular}


modelos (modelo socioambiental integrado y modelo ELM), de forma que el primero genere las condiciones de contorno (cambios de uso del suelo, dinámica agraria y turística, opciones de gestión ambiental...) para el segundo, constituyendo ambos una herramienta de modelado unificada e integrada.

Como un primer paso en la aplicación de este modelo al caso del Mar Menor, se han implementado sólo los módulos hidrológico y de salinidad, ignorando en esta primera fase la realimentación dinámica de los cambios en la vegetación, los nutrientes y el suelo. Posteriores fases en la aplicación del modelo incluirán la adaptación de los módulos biológico y biogeoquímico, modificaciones en distintos módulos y su conexión con el modelado de la dinámica socioeconómica y los cambios de uso del suelo. El módulo hidrológico incluye los flujos verticales de agua y sales entre la atmósfera, el agua superficial, la capa insaturada y la capa subterránea saturada, con interacción explícita entre el agua superficial y subterránea en cada unidad de tiempo. Los flujos horizontales tienen lugar entre el agua superficial y subsuperficial y los flujos de las ramblas, que interaccionan también con el agua subterránea.

ELM fue diseñado para ser explícitamente escalable, por lo que resulta relativamente simple adaptarlo a la extensión y resolución espacial requerida en cada caso. Para la cuenca del Mar Menor se ha utilizado una resolución espacial de $200 \mathrm{~m}$. Como datos de entrada para el modelo figuran diversas capas ráster y vectoriales: el modelo digital de elevaciones, la conductividad hidráulica saturada (estimada a partir de las características de las capas superficiales del suelo y las estimas de Jiménez-Martínez 2010), las condiciones iniciales de agua superficial y de la zona insaturada, el uso del suelo (CORINE Land Cover de 2000, Carreño et al. 2008), la densidad del suelo, el contenido en materia orgánica de la capa superficial, la red de ramblas y los principales canales de distribución del trasvase Tajo-Segura. Otros datos de entrada del modelo incluyen las alturas mareales que inciden en los intercambios entre el Mar Menor y el Mar Mediterráneo, la precipitación diaria y la evapotranspiración potencial, interpolada a partir de los datos aportados por las estaciones meteorológicas de la cuenca. Se han aproximado los parámetros y condiciones iniciales de los distintos usos del suelo a las coberturas más equivalentes en las aplicaciones precedentes del ELM, que incluyen 28 tipos de coberturas, desde pinares con distinta densidad a matorrales y distintos hábitat de saladar y humedal. Para esta primera aplicación se considera sólo la dinámica hidrológica natural ligada a los flujos de precipitación y escorrentía.

\section{Opciones de gestión}

La preocupación por la contaminación en la cuenca del Mar Menor, declarada Zona Vulnerable a la Contaminación por Nitratos, en aplicación de la Directiva de Nitratos (91/676/EEC) y sus efectos sobre la laguna del Mar Menor, declarada Zona Sensible en aplicación de la Directiva de Aguas Residuales (91/271/EEC), ha llevado a plantear distintas medidas para controlar y reducir los flujos de contaminación entre la cuenca y la laguna, entra las que se encuentra i) la aplicación de medidas agroambientales (Consejería de Agricultura, Agua y Medio Ambiente 2003) ; ii) la reutilización de drenajes agrícolas para su uso de nuevo como agua de riego (SACYR 1997); iii) la mejora de los procesos de depuración de aguas residuales en el entorno del Mar Menor, cuyos objetivos incluían el vertido cero al Mar Menor y la plena reutilización de las aguas residuales (DGA 2000) y iv) la utilización de los humedales periféricos del Mar Menor para retener y reducir parte de los nutrientes de origen difuso de la cuenca (Vidal-Abarca y Esteve Selma 1998). De entre tales medidas cabe destacar i) la reutilización de drenajes agrícolas, dado que las infraestructuras necesarias fueron ya ejecutadas (SACYR 1997), aunque no puestas en marcha y ii) la utilización de humedales para la retención de nutrientes de origen difuso, dada la gran cantidad de estudios y experiencias que demuestran el enorme potencial para ello de estos ecosistemas (véase por ejemplo Mitsch y Gosselink 2000; Fisher y Acreman 2004; Alexander et al. 2009). Se ha aplicado el modelo socioambiental integrado para explorar los posibles efec- tos de estas dos medidas, que se describen a continuación con mayor detalle.

La reutilización de drenajes agrícolas, cuyas infraestructuras ha sido ya construidas pero no puestas en funcionamiento, constituye una medida de gestión propuesta por la administración del agua de la cuenca (Confederación Hidrográfica del Segura) con el fin de recoger los drenajes agrícolas procedentes del regadío, impulsarlos hasta una planta desalinizadora y enviarlos de nuevo a los canales de distribución del agua de riego. De esta forma se reutilizarían los drenajes agrícolas de nuevo en regadío, a la vez que se impide la llegada a la laguna de los nutrientes contenidos en los mismos.

Por su parte, la medida de recuperación de humedales consistiría en i) restaurar una parte de la superficie del humedal de Marina del Carmolí perdida por transformación agrícola y que ahora constituye un cultivo marginal y ii) Volver a conectar la rambla del Albujón, actualmente encauzada, con el área de humedal, desencauzando el tramo final de la misma. La rambla del Albujón es el principal cauce de la cuenca, constituyendo la entrada más importante de nutrientes a la laguna. Tras su encauzamiento, los caudales transportados quedaron desconectados del humedal, por lo que reconectar dicha rambla con áreas recuperadas de humedal permitiría que los nutrientes contenidos en sus aguas vuelvan a ser parcialmente retenidos y eliminados antes de llegar a la laguna del Mar Menor.

El modelo socioambiental integrado está elaborado de forma que permite la simulación de las opciones de gestión descritas y de otros escenarios. Cada escenario está definido en el modelo como un vector específico de valores de parámetros.

\section{Resultados}

\section{Modelo socioambiental integrado. Resultados de la simulación}

Los resultados de la simulación del modelo dinámico integrado muestran que el mismo refleja adecuadamente el comportamiento observado para las variables con datos disponibles. El modelo muestra el pronunciado incremento del regadío, favorecido por dos factores: el trasvase Tajo-Segura iniciado en 1979 y la mayor rentabilidad diferencial del regadío frente al secano, especialmente en el caso de los invernaderos (Fig. 6). El incremento del regadío se realiza a expensas de una cierta reducción de la superficie ocupada por vegetación natural y sobre todo por una significativa reducción de la agricultura de secano (Fig. 7). Los nuevos perímetros de riego implican un aumento considerable del aporte de fertilizantes en la cuenca, de forma que el incremento de los drenajes agrícolas lleva aparejado también un mayor movimiento de nutrientes de origen agrícola hacia la laguna del Mar Menor (Fig. 8).

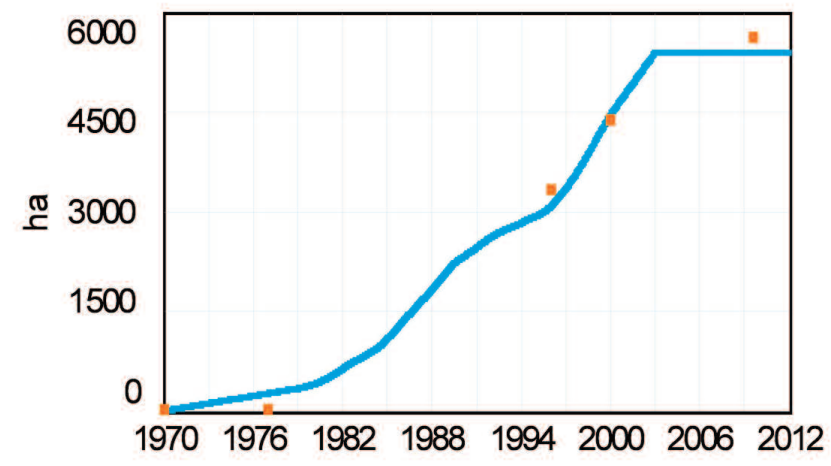

Datos observados (teledetección) | " | "
Simulación del modelo

Figura 6. Evolución de la superficie ocupada por regadío en invernaderos en la cuenca del Mar Menor. Valores observados y simulados con el modelo dinámico. 


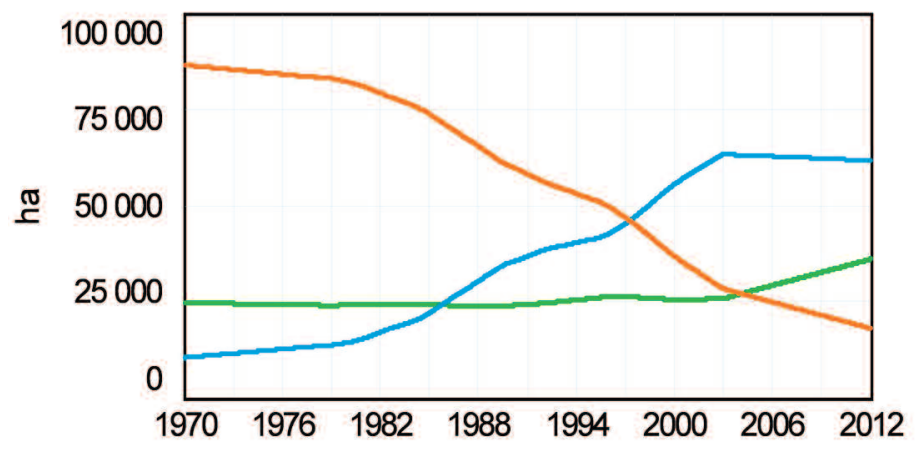

Secano

Regadío

Natural y secano abandonado

Figura 7. Evolución de los usos generales (secano, regadio y natural) en la cuenca del Mar Menor. Resultados de la simulación.

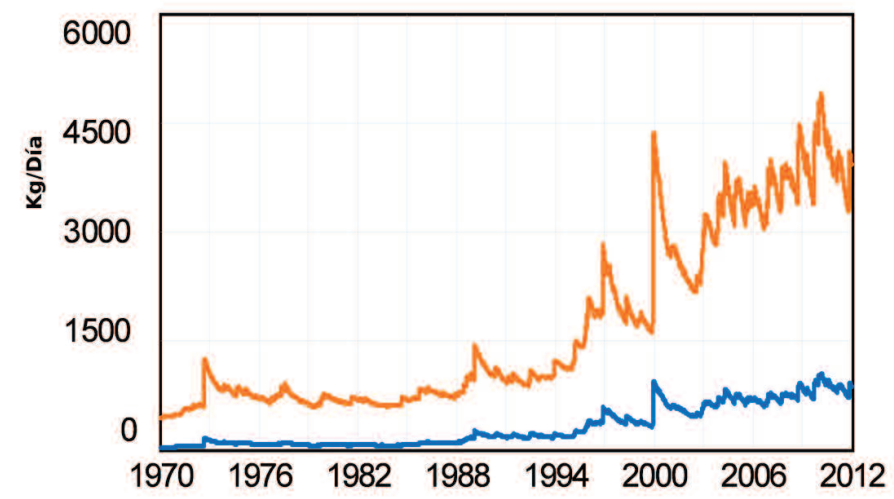

Entrada N de fuentes difusas Entrada $P$ de fuentes difusas

Figura 8. Simulación del patrón de entrada diaria de NID (nitrógeno inorgánico disuelto) y PID (fósforo inorgánico disuelto) de origen difuso a la laguna del Mar Menor. Valores suavizados con media móvil de 365 días.

Por otra parte, la población en el área de influencia del Mar Menor, tanto residente (Fig. 9) como estacional, muestra un rápido crecimiento a lo largo de las últimas décadas debido a la expansión de las actividades turísticas, lo que ha dado lugar al aumento de la exportación de nutrientes de origen urbano. Junto a esta tendencia de aumento en el tiempo, las simulaciones muestran una fuerte dinámica estacional (Fig. 10), con bruscos aumentos de la entrada de nitrógeno y fósforo procedente de las fuentes urbanas durante la época estival.

Si bien no se dispone de series históricas de datos de entrada de nitrógeno y fósforo a la laguna del Mar Menor, los valores estimados de acuerdo con los resultados de la simulación del modelo apuntan a una entrada de nitrógeno al Mar Menor procedente de la cuenca con un promedio en torno a unas 1000 toneladas anuales en el periodo reciente, con grandes fluctuaciones entre 700 y 1600 toneladas anuales. Estas fluctuaciones son generadas fundamentalmente por la gran variabilidad de las precipitaciones y la ocurrencia de episodios de avenida, momento en el que se produce un lavado de grandes cantidades de nutrientes y materiales de la cuenca hacia el Mar Menor.

\section{Aplicación del modelo ELM al Mar Menor. Resultados preliminares}

La Figura 11 muestra una simulación larga (41 años) de la precipitación neta (precipitación menos evapotranspiración real) en la cuenca completa (incluyendo la laguna del Mar Menor), mostrando la gran variabilidad interanual de las precipitaciones. La Figura 12 presenta resultados de la simulación entre los años 2003 y 2010 relativos al nivel relativo del agua en un mes seco y en un mes hú-
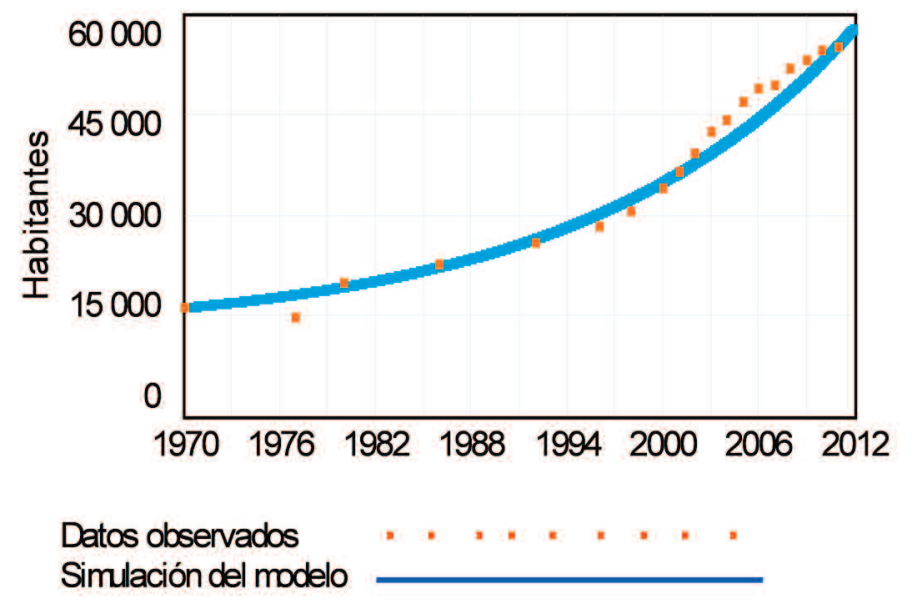

Figura 9. Población residente en el entorno del Mar Menor. Valores observados y simulados con el modelo dinámico

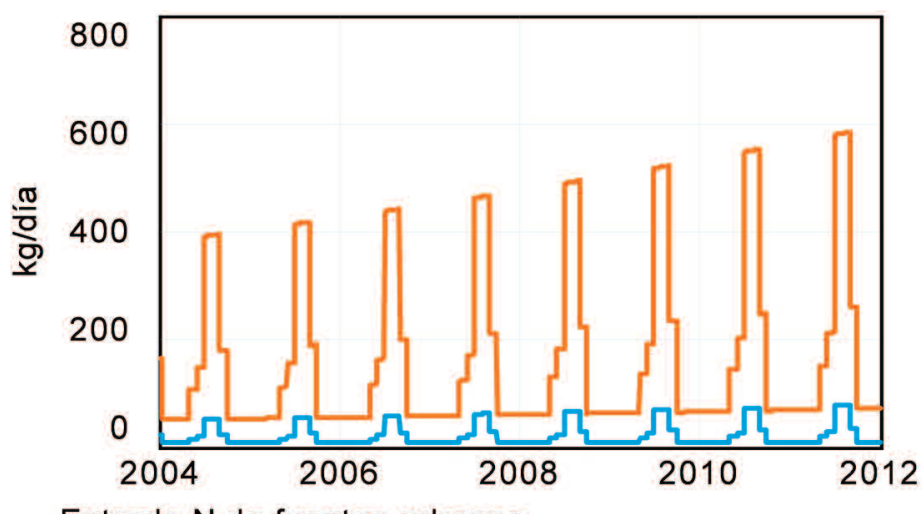

Entrada $\mathrm{N}$ de fuentes urbanas Entrada $\mathrm{P}$ de fuentes urbanas

Figura 10. Dinámica estacional de la entrada de nitrógeno y fósforo a la laguna desde las áreas urbanas del entorno del Mar Menor.

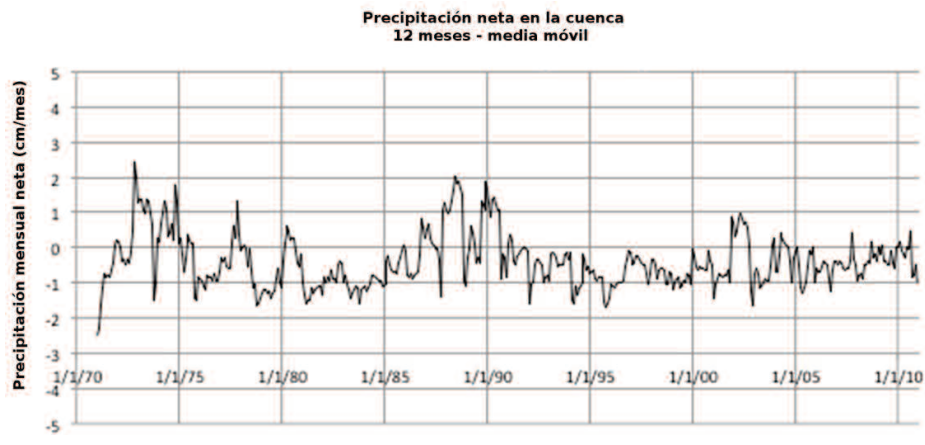

Figura 11. Simulación de la precipitación neta (precipitación menos evapotranspiración real) en la cuenca completa (incluyendo la laguna del Mar Menor) a lo largo de un periodo de 41 años. Valores mensuales suavizados con una media móvil de 12 meses.

medo, periodo para el que existe una mayor disponibilidad de los datos requeridos para la calibración y parametrización del entorno ELM. La figura muestra la importancia de la cuenca del Albujón, que concentra una parte muy significativa del flujo superficial y constituye la principal entrada de agua a la laguna del Mar Menor. Estas simulaciones iniciales, si bien preliminares, parecen mostrar que la aplicación del ELM al Mar Menor puede ayudar a entender los cambios de largo plazo en el funcionamiento hidrológico de la cuenca como respuesta a los cambios de uso del suelo y a cambios ambientales, incluyendo el cambio climático, una vez se implementen las modificaciones pertinentes. 

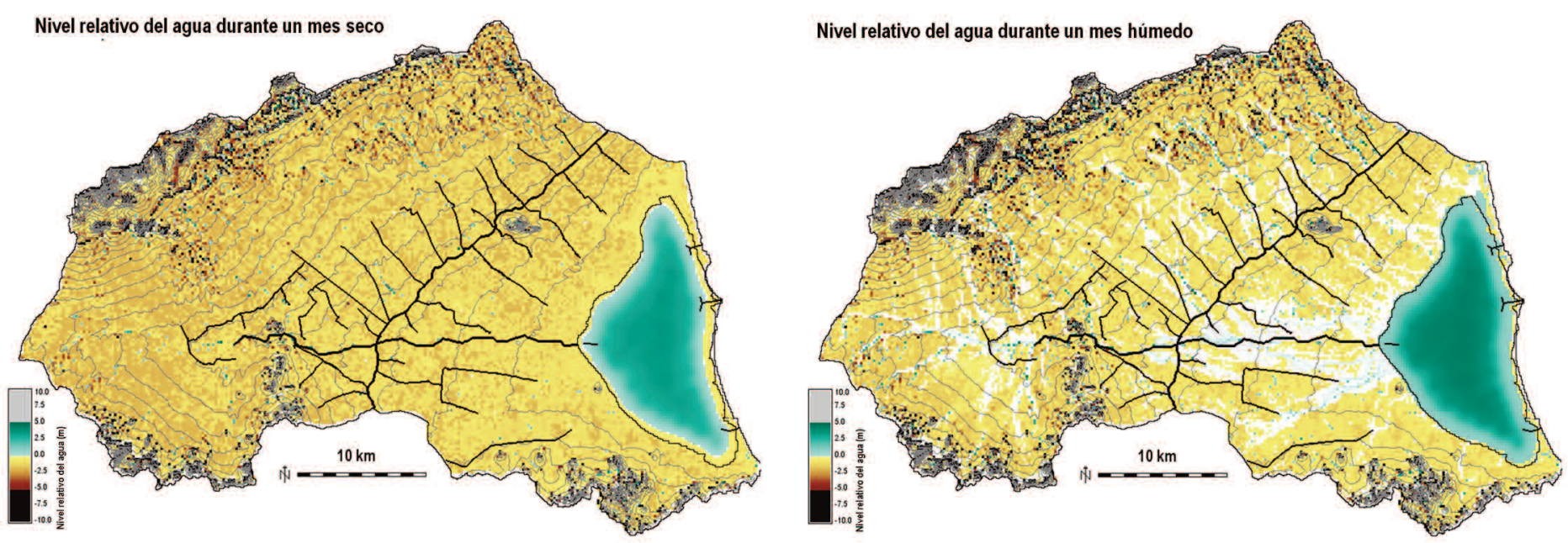

Figura 12. Nivel relativo del agua (respecto a la superficie topográfica) durante un mes húmedo (Enero de 2010). Valores medios diarios de la simulación a lo largo de 8 años (2003-2010) sin considerar las aportaciones del trasvase Tajo-Segura.

\section{Opciones de gestión. Resultados de la simulación}

Se han analizado los efectos esperables de las medidas de gestión de reutilización de drenajes agrícolas y de recuperación de humedales con ayuda del modelo dinámico. La reducción en la entrada final de nutrientes con la medida de reutilización de drenajes sería de alrededor de un $11 \%$ en el caso del nitrógeno procedente de las aguas superficiales y de un $8.5 \%$ si se tienen en cuenta todas las fuentes de entrada de nitrógeno a la laguna (fuentes agrícolas y urbanas). Por su parte, la recuperación del humedal permitiría eliminar el $40 \%$ de los nutrientes de fuentes difusas que llegan a la laguna a través de la rambla del Albujón (Fig. 13).

\section{Discusión}

La cuenca del Mar Menor ha experimentado en las últimas décadas una gran transformación hacia usos más intensivos con la expansión del regadío, a raíz de la puesta en marcha en 1979 del trasvase Tajo-Segura. La rentabilidad diferencial es el factor que ha gobernado los cambios de uso desde los menos intensivos (secano) hacia los más intensivos (regadío herbáceo al aire libre e invernaderos), siguiendo un patrón muy general en el ámbito mediterráneo (Antrop 1993; de Aranzábal et al. 2008). El consiguiente aumento de los flujos hídricos se ha visto confirmado por el ascenso de los niveles piezométricos (Rodriguez Estrella 2009; Aragon et al. 2009) así como por el incremento de los periodos de inundación y el aumento de la humedad edáfica en los humedales del Mar Menor (Alvarez-Rogel et al. 2007). Los resultados obtenidos apuntan al origen mayoritariamente agrario de los nutrientes exportados desde la cuenca, como ocurre en otras cuencas agrícolas intensivas (Jordan et al. 1997; Kronvang 1999; Meissner et al. 2002; Lacroix et al. 2005). Por otra parte, el desarrollo urbanoturístico y los bruscos aumentos de población durante el verano suponen importantes aumentos del consumo de agua y de la producción de aguas residuales. La entrada de nitrógeno y fósforo procedente de los vertidos urbanos ha ido creciendo a lo largo del tiempo, si bien en los últimos años la construcción y mejora de las estaciones depuradoras de aguas residuales ha contrarrestado en parte el efecto del intenso crecimiento de la población.

Las simulaciones aportan unos valores estimados de exportación media interanual de nutrientes a la laguna de unos 8 $\mathrm{kg} / \mathrm{ha}$.año, con un rango entre 6 y $14 \mathrm{~kg} / \mathrm{ha}$.año, valores cercanos a los obtenidos en otras cuencas agrícolas intensivas. Por ejemplo, Mattikalli y Richards (1996) encuentran valores entre 13 y $18 \mathrm{~kg} / \mathrm{ha}$ año; David et al. (1997) entre 20 y 29 kg/ha año y Jordan et al. (1997) encuentran una media para 17 subcuencas de $7 \mathrm{~kg} / \mathrm{ha}$.año, con un rango entre 1 y $16 \mathrm{~kg} / \mathrm{ha}$.año. Los valores de entrada de nitrógeno estimados con el modelo son coherentes con los datos empíricos de concentración de nitrógeno de los principales flujos de la cuenca, los cuales se sitúan entre 28 y $60 \mathrm{mg} / \mathrm{l}$ de nitrógeno inorgánico disuelto (Lloret et al. 2005; Velasco et al. 2006, García Pintado et al. 2009, Serrano y Sironi 2009; Alvarez Rogel et al. 2009) y con la estima de entrada media anual de agua a la laguna, que sobre la base del trabajo de Senent Alonso et al. (2009), puede

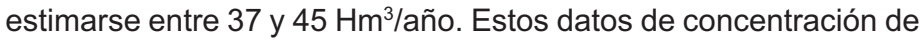
nitrógeno y las estimas de descarga anual media de agua al Mar Menor conjuntamente aportan una estima de entrada anual media de nitrógeno a la laguna situada entre 1000 y 1300 toneladas anuales, rango similar al obtenido con el modelo dinámico.

La elaboración y aplicación de un modelo socio ambiental específico para la cuenca del Mar Menor permite incorporar con fidelidad las características y procesos singulares de dicha cuenca e integrar en el propio modelo la estructura y variables requeridas para simular los escenarios y opciones de gestión de interés. En este trabajo se han presentado los resultados relativos a las principales de tales medidas: la reutilización de drenajes agrícolas y la recuperación de humedales. La medida de recuperación de humedales representaría una reducción del $40 \%$ en la entrada de nutrientes aportados a través de la subcuenca del Albujón, duplicando la reducción que se obtendría en esta subcuenca con la reutilización de drenajes. Aunque esta medida se refiere solamente a dicha subcuenca, los resultados son significativos, dado que la rambla del Albujón es la principal fuente de contaminación de origen agrí-

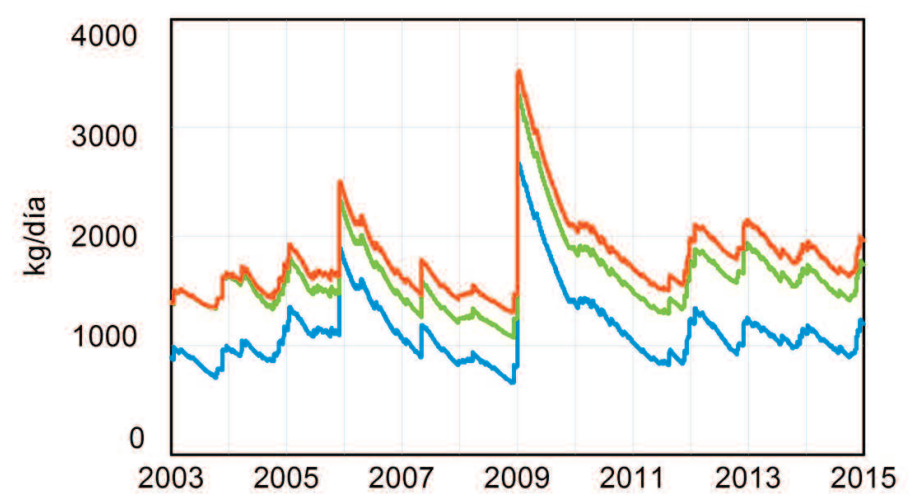

Simulación base

Reutilización de drenajes

Recuperación de humedales

Figura 13. Entrada diaria de nitrógeno de origen difuso desde la subcuenca del Albujón hacia la laguna del Mar Menor bajo la simulación base y bajo las opciones de gestión de reutilización de drenajes y de recuperación de humedales. 
cola en la laguna, no sólo en relación con los nutrientes sino en relación a otros componentes como los pesticidas (Campillo et al. 2013; Moreno-González et al. 2013), los cuales están afectando a las comunidades de bivalvos del Mar Menor (Campillo et al. 2013).

Además, un análisis coste-efectividad (es decir, de comparación del grado de consecución de ciertos objetivos por cada unidad monetaria invertida bajo distintas medidas de gestión) aplicado para evaluar la efectividad relativa de cada una de tales medidas, ha mostrado que la recuperación de humedales es una medida no sólo más eficaz que la reutilización de drenajes sino también económicamente más eficiente, dado que por cada 100 euros invertidos en la medida de reutilización de drenajes se consiguen retirar $8.1 \mathrm{~kg}$ de nitrógeno y $2.9 \mathrm{~kg}$ de fósforo, mientras que ese mismo gasto empleado en la recuperación del humedal retira prácticamente el doble de ambos nutrientes: $16.2 \mathrm{~kg}$ de nitrógeno y $5.7 \mathrm{~kg}$ de fósforo (Martínez-Paz et al. 2007). Estos valores se sitúan en la línea de otros estudios que muestran que la restauración de humedales es una opción claramente coste-efectiva (Gren et al. 1997; Turner et al. 1999; Gustafson et al. 2000; Zanou et al. 2003, Lacroix et al. 2005). Lacroix et al. (2005) también señala que aunque a veces se considera la restauración de humedales como una medida costosa, en realidad suele ser más económica que otras, como los subsidios para la reducción de la aportación de fertilizantes. Estos resultados son relevantes, dado que con anterioridad no se disponía de evaluación alguna de la eficacia ni la efectividad de la medida de reutilización de drenajes, pese a que todas las infraestructuras necesarias (red de canales de drenaje, impulsiones y planta desaladora, entre otros) fueron ya construidas por la administración del agua (Confederación Hidrográfica del Segura).

Se ha señalado que de forma general los modelos y sus resultados no pueden transferirse sin una reconsideración de sus asunciones, estructura y propósito (Galic et al. 2013), apuntando a la importancia de atender el contexto específico. Los resultados expuestos muestran la utilidad y relevancia de disponer de herramientas de modelización integrada como la aplicada en el caso del Mar Menor, donde la incorporación de los factores socioeconómicos, la consideración explícita del contexto socioeconómico y ambiental específico de la cuenca bajo estudio y la utilización de una perspectiva a largo plazo permite simular los escenarios y opciones de gestión concretas que son de interés para gestores y agentes y responder por tanto a preguntas clave en los procesos de planificación y de toma de decisiones. Sin embargo, una comprensión más profunda de la dinámica de la cuenca requiere aplicar modelos dinámicos que incorporen de forma explícita la dimensión espacial, como el entorno ELM, cuya aplicación al Mar Menor está en fase de desarrollo.

\section{Agradecimientos}

El presente trabajo ha sido parcialmente financiado con el contrato: "Modelización integrada para la gestión sostenible del Mar Menor", elaborado para TRAGSA (2011-2012) y cuyo soporte es agradecido.

\section{Referencias}

Alexander, R.B., Böhlke, J.K., Boyer, E.W., David, M.B., Harvey, J.W., Mulholland, P.J., Seitzinger, S.P., Tobias, C.R., Tonitto, C., Wollheim, W.M. 2009. Dynamic modelling of nitrogen losses in river Networks unravels the coupled effects of hydrological and biogeochemical processes. Biogeochemistry 93: 91-116.

Alvarez-Rogel, J., Jimenez-Carceles, F.J., Roca, M.J., Ortiz, R., 2007. Changes in soils and vegetation in a Mediterranean coastal salt marsh impacted by human activities. Estuarine, Coastal and Shelf Science 73: 510-526.

Álvarez-Rogel, J., Jiménez-Cárceles, F.J., Egea Nicolás, C., María-Cervantes, A., González-Alcaraz, M.N., Párraga Aguado, I., Conesa Alcaraz, H.M. 2009. Papel de los humedales costeros del Mar Menor en la depuración de aguas eutrofizadas: el caso de la Marina del Carmolí. En: Cabezas, F., Senent, M. (eds.), Mar Menor. Estado actual del conocimiento científico, pp. 321-358. Fundación Cluster-Instituto Euromediterráneo del agua. Murcia, España.
Antrop, M. 1993. The transformation of the Mediterranean landscapes: an experience of 25 years of observations. Landscape and Urban Planning 24: 3-13.

Aragon, C., Jiménez-Martínez, J., Gaqrcía-Aróstegui, J.L., Hornero, J. 2009. Hidrogeología y recursos subterráneos en el área Campo de Cartagena-Mar Menor. En: Cabezas, F., Senent, M. (eds.), Mar Menor. Estado actual del conocimiento científico, pp. 85-108, Fundación Cluster-Instituto Euromediterráneo del agua. Murcia, España.

Campillo, J.A., Albentosa, M., Valdés, J., Moreno-González, R., León, V. 2013. Impact assessment of agricultural inputs into a Mediterranean costal lagoon on transplanted clams (Ruditapes decussates) by biochemical an physiological responses. Aquatic Toxicology 142-143, 365379.

Castro Díez, P. 1996. Variaciones estructurales y funcionales de los fanerófitos dominantes en las comunidades de encinar a lo largo de un gradiente climático atlántico-mediterráneo. Tesis Doctoral. Universidad de León. León, España.

Carreño, M.F., Esteve, M.A., Martinez, J., Palazón, J.A., Pardo, M.T. 2008. Habitat changes in coastal wetlands associated to hydrological changes in the watershed. Estuarine. Coastal and Shelf Science 77, 475-483.

Chapelle, A., Duarte, P., Fiandrino, A., Esteve, M.A., Galbiati, L., Marinov, D., et al. 2005. Comparison between different modelling approaches for coastal lagoons. Institute for Environment and Sustainability. Ispra. Joint Research Centre. European Commission. Report EUR 21817 EN.

Conesa, H., Jiménez-Cárceles, F.J. 2007. The Mar Menor lagoon (SE Spain): A singular natural ecosystem threatened by human activities. Marine Pollution Bulletin 54, 839-849.

Consejería de Agricultura, Agua y Medio Ambiente 2003. Código de Buenas Prácticas Agrarias en la Región de Murcia. Comunidad Autónoma de la Región de Murcia. Murcia. España.

David, M.B., Gentry, L.E. Kovacic, D.A. Smith, K.M. 1997. Nitrogen balance in and export from an agricultural watershed. Journal of Environmental Quality 26: 1038-1048.

DGA 2000. Plan de Saneamiento y Depuración de las Aguas Residuales Urbanas de la Región de Murcia (2001-2010). Dirección General del Agua. Comunidad autónoma de la región de Murcia. Murcia. España.

de Aranzábal, I., Schmitz, M.F., Aguilera, P., Díez Pineda, F. 2008. Modelling of landscape changes derived from the dynamics of socio-ecological systems A case of study in a semiarid Mediterranean landscape. Ecological Indicators 8: 672-685.

EEA 2010. Ecosystem accounting and the cost of biodiversity losses. The case of coastal Mediterranean wetlands. EEA Technical report $\mathrm{N}^{\circ}$ 3/2010. Office for Official Publications of the European Union. Luxembourg.

Esteve, M.A., Carreño, M.F., Robledano, F., Martínez-Fernández, J., Miñano, J. 2008. Dynamics of coastal wetlands and land use changes in the watershed: implications for the biodiversity. En: Russo, R.E. (Ed.): Wetlands: Ecology, Conservation and Restoration, pp. 133-175. Nova Science Publishers. New York. USA.

Fisher, J., Acreman, M.C. 2004. Wetland nutrient removal: a review of the evidence. Hydrology and Earth System Sciences 8: 673-685.

Fitz, H.C. 2008. Documentation of the Everglades Landscape Model: Application of ELMwca1 v2.8. Final report submitted to South Florida Water Management District, Everglades Division. West Palm Beach, FL. USA. Disponible en: http://ecolandmod.ifas.ufl.edu/publications/

Fitz, H.C. 2010. Ecological landscape modeling: the general application of an existing simulation framework. EDIS Document SL321, Soil and Water Science Department, IFAS, University of Florida. Gainesville, FL. USA. Disponible en: http://edis.ifas.ufl.edu/ss532.

Fitz, H.C., Kiker, G.A., Kim, J.B. 2011. Integrated ecological modeling and decision analysis within the Everglades landscape. Critical Reviews in Environmental Science and Technology 41:517 - 547. Disponible en: http://ecolandmod.ifas.ufl.edu/publications/

Fitz, H.C., Paudel, R. 2012. Documentation of the Everglades Landscape Model: ELM v2.8.4. Ft. Lauderdale Research and Education Center, University of Florida. Gainesville, FL. USA. Disponible en: http://ecolandmod.ifas.ufl.edu/publications/

Galic, N., Schmolke, A., Forbes, V., Baveco, H., van den Brink, P.J. 2012. The role of ecological models in linking ecological risk assessment to ecosystem services in agroecosystems. Science of the Total Environment 415, 93-100.

García Pintado, J., Barberá, G., Martínez-Mena, M., Albaladejo, J., Erena, M., Castillo, V. 2009. Caracterización y evaluación de fuentes antrópicas de nutrientes transportados por la rambla del Albujón hacia el Mar 
Menor. En: Cabezas, F., Senent, M. (eds.), Mar Menor. Estado actual del conocimiento científico, pp. 167-206. Fundación Cluster-Instituto Euromediterráneo del agua. Murcia. España.

Gómez Cerezo, R. 1995. Función de los humedales en la dinámica de nutrientes $(N$ y $P$ ) de una cuenca de características áridas: Experiencias en el Sureste Ibérico. Tesis Doctoral. Universidad de Murcia. Murcia, España.

Gren, I.M., Elofsson, K., Jannke, P. 1997. Cost-effective nutrient reductions to the Baltic Sea. Environmental and Resource Economics 10, 341-362.

Guimera, J., Marfà, O., Candela, L., Serrano, L. 1995. Nitrate leaching and strawberry production under drip irrigation management. Journal of Environmental Quality 56 : 121-135.

Gustafson, A., Fleischer, S., Joelsson, A. 2000. A catchment-oriented and cost-effective policy for water protection. 2000. Ecological engineering $14,419-427$

Houlahan, J.E., Findlay, C.S., 2004. Estimating the 'critical' distance at which adjacent land-use degrades wetland water and sediment quality. Landscape Ecology 19, 677-690.

IUCN 2002. Integrated Water Management to Address Environmental Degradation in the Mediterranean Region. IUCN Mediterranean office. Málaga. España.

Jiménez-Martínez, J. 2010. Aquifer recharge from intensively irrigated farmland. Several approaches. Technical University of Catalonia. Barcelona, España.

Jordan, E., Correll, D., Weller D. 1997. Effects of agriculture on Discharges of Nutrients from Coastal Plain Watersheds of Chesapeake Bay. Journal of Environmental Quality 26: 836-848.

Kelly, R.A., Jakeman, A.J., Barreteau, O., Borsuk, M.E., ElSawah, S., Hamilton, S.H., Henriksen, H.J., Kuikka, S., Maier, H.R., Rizzoli, A.E., van Delden, H., Voinov, A. 2013. Selecting among five common modelling approaches for integrated environmental assessment and management. Environmental Modelling y Software 47 (2013) 159-181.

Kronvang, B., Svendsen, L.M., Jensen, J.P., Dørge, J. 1999. Scenario analysis of nutrient management at the river basin scale. Hydrobiologia 410: 207-212.

Lacroix, A., Beaudoin, B., Makowsk, D. 2005. Agricultural water nonpoint pollution control under uncertainty and climate variability. Ecological Economics 53: 115- 127.

Lloret, J., Marin, A., Marin-Guirao, L., Velasco, J. 2005. Changes in macrophytes distribution in a hypersaline coastal lagoon associated with the development of intensively irrigated agriculture. Ocean y Coastal Management 48, 828-842.

Lloret, J., Marin, A., Marin-Guirao, L. 2008. Is coastal lagoon eutrophication likely to be aggravated by global climate change?. Estuarine, Coastal and Shelf Science 78, 403-412.

Maltby, E., Acremanb, M., Blackwellc, M.S.A., Everardd, M., Morrise, J. 2013. The challenges and implications of linking wetland science to policy in agricultural landscapes - experience from the UK National Ecosystem Assessment. Ecological Engineering 56, 121- 133.

Martínez Fernández, J., Esteve Selma, M.A. 2003. El papel de las aguas subterráneas en la exportación de nutrientes de origen agrícola hacia la laguna del Mar Menor. En: Fornés, J.M., Llamas, M.R. (eds.): Conflictos entre el desarrollo de las aguas subterráneas y la conservación de humedales: litoral mediterráneo, pp. 191 - 213. Fundación Marcelino Botín-Ediciones Mundi- Prensa. Madrid. España.

Martínez Fernández, J., Martínez Fernández, J., López Bermúdez, F., Belmonte Serrato, F. 1994. Crecimiento y producción primaria de Rosmarinus officinalis en relación con algunos factores ambientales. Ecologia 8: $177-183$.

Martínez-Fernández, J., López-Bermúdez, F., Martínez-Fernández, J., Romero-Díaz, A. 1995. Land Use and Soil-Vegetation Relationships in a mediterranean ecosystem: El Ardal, Murcia (Spain). Catena 25: 153-167.

Martínez Fernández, J., Martínez Fernández, J., Romero Díaz, M.A., López Bermúdez, F., Belmonte Serrato, F. 1996. Biomasa e índice foliar de Rosmarinus officinalis en matorral semiárido (Cuenca de Mula, Murcia). Anales de Biología 21: 83-92.

Martínez-Fernández, J., Esteve-Selma, M.A. 2004. Dynamics of water scarcity on irrigated landscapes: Mazarron and Aguilas in south-eastern Spain. System Dynamics Review 20: 117-137.

Martínez-Fernández, J., Esteve-Selma, M.A., Calvo Sendín, J.F. 2000. Environmental and socioeconomical interactions in the evolution of traditional irrigated lands: a dynamic system model. Human Ecology 28: 279-299.
Martínez-Fernández, J., Esteve Selma, M.A., Baños-González, I., Carreño, F., Moreno, A. 2013. Sustainability of Mediterranean irrigated agrolandscapes. Ecological Modelling 248: 11-19.

Martínez-Fernández, J., Esteve-Selma, M.A.;, Martínez-Paz, J.M., Carreño, M.F., Martínez-López, J., Robledano, F., Farinós, P. (en prensa). Tradeoffs between Biodiversity Conservation and Nutrients Removal in Wetlands of arid Intensive Agricultural Basins: the Mar Menor case, Spain. En: Jørgensen, S.E., N-B. Chang, N-B., Xu, F-L. (eds.): Advances in Ecological Modelling and Ecological Engineering applied on Lakes and Wetlands. Elsevier. USA.

Martinez Paz, J.M., Martinez Fernández, J., Esteve Selma, M.A. 2007. Evaluación económica del tratamiento de drenajes agrícolas en el Mar Menor (SE España). Revista Española de Estudios Agrosociales y Pesqueros 215/216. 211-231.

Mattikalli, N., Richards, K. 1996. Estimation of Surface Water Quality Changes in Response to Land Use Change: Application of The Export Coefficient Model Using Remote Sensing and Geographical Information System. Journal of Environmental Management 48: 263-282.

Mattos, D., Quaggio, J.A., Cantarella, H., Kumar Alva, A. 2003. Nutrient content of biomass components of Hamlin sweet orange trees. Scientia Agricola 60(1), 155-160.

Meissner, R., Seeger, J., Rupp, H. 2002. Effects of agricultural land use changes on diffuse pollution of water resources. Irrigation and Drainage 51: 119-127.

Mitsch, WJ., Gosselink, J.G. 2000. Wetlands. John Wiley and Sons. New York. USA.

Moreno, F., Cayuela, J.A., Fernandez, J.E., Fernández-Boy, E., Murillo, J.M., Cabrera, F. 1996. Water Balance and nitrate leaching in an irrigated maize crop in SW Spain. Agricultural Water Management 32: 71-83.

Moreno-González, R., Campillo, J.A., García, V., León, V.M. 2013. Seasonal input of regulated and emerging organic pollutants through surface watercourses to a Mediterranean coastal lagoon. Chemosphere. http://dx.doi.org/10.1016/j.chemosphere.2012.12.022

Nemec, K., Raudsepp-Hearne, C. 2013. The use of geographic information systems to map and assess ecosystem services. Biodiversity and Conservation 22, 1-15.

NRC 2012. Progress Toward Restoring the Everglades: The Fourth Biennial Review, 2012. National Academies Press, Washington, DC. USA.

Olmo Conesa, F. 2004. Estudio de los aportes de fertilizantes en el Campo de Cartagena. Evolución en el tiempo y situación actual. Proyecto Fin de Carrera. Universidad de Murcia. Murcia.

Pardo, M.T., Esteve, M.A., Giménez, A., Martínez-Fernández, J., Carreño, M.F, Serrano, J., Minaño, J.2008. Assessment of the hydrological alterations on wandering beetle assemblages (coleoptera: Carabidae and Tenebrionidae) in coastal wetlands of arid Mediterranean systems. Journal of Arid Environments 72, 1805-1810.

Perez Ruzafa, A., Gilabert, J., Gutiérrez, J.M., Fernández, A.I., Marcos, C., Sabah, S. 2002. Evidence of a planktonic food web response to changes in nutrient input dynamics in the Mar Menor coastal lagoon, Spain. Hydrobiologia 475/476, 359-369.

Ramirez Santigosa, I., Vicente Albaladejo, M., García Barceló, J.A., Vaquero Gómez, A. 1999. Mapa digital de suelos de la Región de Murcia. Consejería de Agricultura, Agua y Medio Ambiente. Murcia (CD-Rom).

Robledano, F., Esteve, M.A., Farinós, P., Carreño, M.F., Martínez, J. 2010. Terrestrial birds as indicators of agricultural-induced changes and associated loss in conservation value of Mediterranean wetlands. Ecological Indicators 10, 274-286.

Robledano, F., Esteve, M.A., Martínez-Fernández, J., Farinos, P. 2011. Determinants of wintering waterbird changes in a Mediterranean coastal lagoon affected by eutrophication. Ecological Indicators 11. 395-406.

Rodríguez Estrella, T. 2009. El Mar Menor: Geología y sus relaciones con las aguas subterráneas del continente. En: Cabezas, F., Senent, M. (eds.), Mar Menor. Estado actual del conocimiento científico, pp. 47-84. Fundación Cluster-Instituto Euromediterráneo del agua. Murcia, España.

Rubio Lopez, J.A. 1999. Incidencia de la política de la Unión Europea en materia de residuos en el sector agrario de la Region de Murcia. Tesis Doctoral. Universidad de Murcia. . España.

SACYR SA. 1997. Análisis y caudales de los drenajes y ramblas del campo de Cartagena. Informe técnico. Confederación Hidrográfica del Segura, Murcia. España

Senent Alonso, M., Martínez Vicente, D., Cabezas, F., García Aróstegui, J.L., Baudron, P. 2009. Aproximación mediante modelización matemática a la 
evaluación de las descargas del acuífero cuaternario del Campo de Cartagena al Mar Menor (Murcia). En: Cabezas, F., Senent, M. (eds.), Mar Menor. Estado actual del conocimiento científico, pp. 109-130. Fundación Cluster-Instituto Euromediterráneo del agua. Murcia, España.

Serrano, J.F., Sironi, J.S. 2009. Cuantificación y evolución de la carga contaminante de nutrientes y plaguicidas en aguas del Mar Menor y su relación con los aportes hídricos de la Rambla del Albujón y otros aportes subterráneos. En: Cabezas, F., Senent, M. (eds.), Mar Menor. Estado actual del conocimiento científico, pp. 245-284. Fundación Cluster-Instituto Euromediterráneo del agua. Murcia, España.

Shah, K.H., Siddiqui, S.H., Memon, M.Y., Aslam, M., Imtiaz, M. Khan, P. 2003. Performance of Fertigation Technique for $P$ Usage Efficiency in Wheat. Asian Journal of Plant Sciences 2 (15-16): 1088-1091.

Shen, Y, Gao, Ch, Nan, Z.B., Bellotti, W. D., Chen, W., Zou, Y., Unkovich, M., Robertson, M.J., Probert, M.E. 2004. Yield and N content response of winter wheat to 2 fallow lengths after 4 years of lucerne in the Gansu Loess Plateau, China. En: New Directions for a Diverse Planet : Proceedings of the 4th International Crop Science Congress, 26 September - 1 October 2004, Brisbane, Australia. Regional Institute. Gosford. Australia.

Turner, K, Georgiou, S., Green, I.M., Wulff, F., Barret, S., Soderqviest, T., Bateman, I., Folke, C., Langaas, S., Zylicz, T., Maler, K.G., Markowska, A.
1999. Managing nutrient fluxes and pollution in the Baltic: an interdisciplinary simulation study. Ecological economics 30(2): 333-352.

Velasco, J., LLoret, J., Millan, A.; Marin, A., Barahona, J., Abellán, P., Sánchez-Fernández, D. 2006. Nutrient and particulate inputs into the Mar Menor lagoon (SE Spain) from an intensive agricultural watershed. Water, Air and Soil Pollution 176: 37-56.

Vidal-Abarca, M.R., Esteve-Selma, M.A. (Coords). 1998. Análisis de viabilidad del uso de humedales para mitigar el efecto de los vertidos y drenajes agrícolas al Mar Menor. Confederación Hidrográfica del Segura, Murcia. España.

Vidal, B., Martínez-Fernández, J., Sánchez Picón, A., Pugnaire, F. 2013. Trade-offs between maintenance of ecosystem services and socio-economic development in rural mountainous communities in southern Spain: a dynamic simulation approach. Journal of Environmental Management 131: 280-297.

Xue, Y., David, M.B., Gentry, L.E., Kovacic, D.A. 1998. Kinetics and modelling of dissolved phosphorous export from a tile-drained agricultural watershed. Journal of Environmental Quality 27, 917-922.

Zanou, B., Kontogianni, A., Skourtos, M. 2003. A classification approach of cost effective managent measures for the improvement of watershed quality. Ocean and Coastal Management 46, 957-983. 